\title{
A Small-Molecule Compound Selectively Activates K2P Channel TASK-3 by Acting at Two Distant Clusters of Residues $\$$
}

\author{
Fuyun Tian, Yunguang Qiu, Xi Lan, Min Li, Huaiyu Yang, and Zhaobing Gao
}

CAS Key Laboratory of Receptor Research, State Key Laboratory of Drug Research, Shanghai Institute of Materia Medica, Chinese Academy of Sciences, Shanghai, China (F.T., Y.Q., X.L., Z.G.); University of Chinese Academy of Sciences, Beijing, China (F.T., Y.Q., X.L., Z.G.); Department of Neuroscience, High Throughput Biology Center and Johns Hopkins lon Channel Center, School of Medicine, Johns Hopkins University, Baltimore, Maryland (M.L.); and Shanghai Key Laboratory of Regulatory Biology, Institute of Biomedical Sciences, School of Life Sciences, East China Normal University, Shanghai, China (H.Y.)

Received November 27, 2018; accepted April 17, 2019

\section{ABSTRACT}

The TASK-3 channel is a member of the K2P family that is important for the maintenance of the resting membrane potential. Previous studies have demonstrated that the TASK-3 channel is involved in several physiologic and pathologic processes, including sleep/wake control, cognition, and epilepsy. However, there is still a lack of selective pharmacological tools for TASK-3, which limits further research on channel function. In this work, using a high-throughput screen, we discovered that $\mathrm{N}$-(2-((4-nitro-2-(trifluoromethyl)phenyl)amino)ethyl)benzamide (NPBA) showed excellent potency and selectivity as a novel TASK-3 activator. The molecular determinants of NPBA activation were then investigated by combining chimera and mutagenesis analysis. Two distant clusters of residues located at the extracellular end of the second transmembrane domain (A105 and A108) and the intracellular end of the third transmembrane domain (E157) were found to be critical for NPBA activation. We then compared the essentials of the actions of NPBA with inhalation anesthetics that nonselectively activate TASK-3 and found that they may activate TASK-3 channels through different mechanisms. Finally, we transplanted the three residues A105, A108, and E157 into the TASK-1 channel, which resists
NPBA activation, and the constructed mutant TASK-1(G105A, V108A, A157E) showed dramatically increased activation by NPBA, confirming the importance of these two distant clusters of residues.

\section{SIGNIFICANCE STATEMENT}

TASK-3 channels conduct potassium and are involved in various physiological and pathological processes. However, the lack of selective modulators has hindered efforts to increase our understanding of the physiological roles of TASK-3 channels. By using a high-throughput screen, we identified NPBA as a potent and selective TASK-3 activator, and we show that NPBA is a more potent activator than terbinafine, the only reported TASK-3 selective activator to date. We also show here that NPBA has outstanding selectivity for TAS-3 channels. These characteristics make NPBA a promising pharmacological probe for research focused on defining TASK-3 channel function(s). In addition, we identified two distant clusters of residues as determinants of NPBA activation providing new molecular clues for the understanding of the gating mechanism of K2P channels.

\section{Introduction}

Potassium channel subfamily K member 2 (K2P) channels conduct "leak" potassium currents, which play critical roles in the maintenance of the resting membrane potential. The K2P family currently has 15 mammalian members, which can be divided into six distinct subfamilies on the basis of structure and functional properties. The tandem pore domain, weak inward-rectifying $\mathrm{K}^{+}$channel (TWIK)-related acid-sensitive $\mathrm{K}$ (TASK)-3 channel is a member of the K2P family and belongs to the TASK subfamily consisting of TASK-3, TASK-1, and

This work was supported by the National Science Fund of Distinguished Young Scholars (81825021), the National Natural Science Foundation of China (81773707, 61433017), and the Strategic Leading Science and Technology Projects of Chinese Academy of Sciences (XDA12040211, XDA12050308).

https://doi.org/10.1124/mol.118.115303.

S This article has supplemental material available at molpharm. aspetjournals.org.
TASK-5 (Enyedi and Czirjak, 2010). TASK-3 and TASK-1 are functional channels conducting currents highly sensitive to extracellular pH (Duprat et al., 1997; Kim et al., 2000; Rajan et al., 2000), whereas TASK-5 cannot be functionally expressed (Kim and Gnatenco, 2001).

Mainly expressed in the central nervous system, particularly in the cerebellum, hypothalamus, and cortex (Talley et al., 2001), TASK-3 was proposed to be related to mood disorders, sleep/wake control, and cognition (Linden et al., 2007; Gotter et al., 2011). The human TASK-3 mutation G236R has been identified as responsible for mental retardation associated with a rare maternally transmitted dysmorphism syndrome (Barel et al., 2008), and TASK-3 knockout mice showed impaired working memory and altered circadian rhythms (Linden et al., 2007). Abundant TASK-3 expression was also found in the adrenal cortex, and it has been demonstrated that TASK-3 channel deletion in mice could

ABBREVIATIONS: Ct, carboxyl terminus; HTS, high-throughput screening; K2P, potassium channel subfamily K member 2; NPBA, N-(2-((4-nitro2-(trifluoromethyl)phenyl)amino)ethyl)benzamide; Trek-1, potassium channel subfamily K member 2; TASK, TWIK-related acid-sensitive K; TM, transmembrane domain; TWIK, tandem pore domain, weak inward-rectifying $\mathrm{K}^{+}$channel. 
recapitulate low-renin essential hypertension (Guagliardo et al., 2012). In addition, TASK-3 has been implicated in other disorders, including cancer (Mu et al., 2003), primary hyperaldosteronism (Davies et al., 2008), and epilepsy (Holter et al., 2005).

The TASK-3 channel, which is involved in multiple physiologic and pathologic processes, can be modulated by various endogenous neurochemicals, clinically active drugs, and physicochemical factors (Goldstein et al., 2001; Lesage, 2003; Talley et al., 2003). The TASK-3 channel is closed by extracellular acidification (Duprat et al., 1997; Kim et al., 2000; Rajan et al., 2000) and it is also inhibited by hormones and transmitters through $\mathrm{G}$ protein-coupled receptors (GPCRs) (Talley and Bayliss, 2002; Chemin et al., 2003; Mathie, 2007); this inhibition is now considered to be mediated by diacylglycerol (Wilke et al., 2014). Moreover, the TASK-3 channel is an important target of inhalation anesthetics (e.g., chloroform) and respiratory stimulants (e.g., doxapram) (Cotten et al., 2006). In addition, the TASK3 channel is sensitive to other small-molecule compounds, such as ruthenium red (Czirják and Enyedi, 2002), anandamide (Berg et al., 2004), lidocaine (Kim et al., 2000), and bupivacaine (Meadows and Randall, 2001). However, all the small-molecule regulators above are nonselective, which limits their use as selective pharmacological tools for research on TASK-3. Currently, the only reported selective activator of TASK-3 is terbinafine, which has moderate potency with a maximum effect of approx. 2-fold and a $\mathrm{pEC}_{50}$ of $6.2 \mu \mathrm{M}$ in the thallium flux assay, but the mechanism of action of this compound remains unknown (Wright et al., 2017).

In mechanistic research on TASK-3 activators, the mechanism of action of inhalation anesthetics on TASK-3 channels has been extensively studied and partially revealed. Specifically, M159, a residue located on the cytoplasmic side of transmembrane domain (TM) 3, was proposed to be the potential binding site of inhalation anesthetics (AndresEnguix et al., 2007), and the reliability of this conclusion was further evidenced by a later study using cysteine modification (Conway and Cotten, 2012). In addition, amino acids 243-248 (VLRFLT), located in the junction of TM4 and the carboxyl terminus (Ct), were also identified as essential for activation by inhalation anesthetics (Patel et al., 1999), and this region was also demonstrated to be important for the regulation of GPCRs or other intracellular factors (Talley and Bayliss, 2002). Several studies on TREK-1 (K2P2.1) concluded that the TM4-Ct junction was critical for the conduction of conformational transmission from the $\mathrm{Ct}$ to the pore of the channel, which could be disturbed by a triple glycine mutation, K2P2.1-3G (Bagriantsev et al., 2011, 2012). The up and down states of TM4 have been proven to represent different conductive states that involve the conformational transmission (Dong et al., 2015).

In this study, we searched for better small-molecule activators of TASK-3 by high-throughput screening (HTS) and identified NPBA as a novel selective agonist of the TASK-3 channel. Furthermore, by adopting various mutagenesis analyses, we found three residues, A108, A105, and E157 that were important for NPBA activation. In addition, we found that the selective activator we identified shared some common key residues with inhalation anesthetics but may conduct channel activation through a different mechanism.

\section{Materials and Methods}

Plasmid Construction. Human TASK-3, human TASK-1, and human TRESK were gifts from Dr. Min Li (Johns Hopkins University). Enhanced green fluorescent protein (EGFP), human TREK-1, and rat THIK1 were provided by Jia Li (Shanghai Institute of Materia Medica, China), Yang Li (Shanghai Institute of Materia Medica), and Haijun Chen (State University of New York). Point mutations of TASK channels were introduced using the QuikChange II Sitedirected Mutagenesis Kit (Stratagene), and chimeras of the TASK channel were constructed using the In-Fusion HD Cloning Kit (TaKaRa). All constructs were verified by sequencing.

Cell Culture and Transient Transfection. Chinese hamster ovary (CHO)-K1 cells were cultured in DMEM/F12 (Gibco) with 10\% FBS (Gibco). Twenty-four hours prior to transfection, the cells were split into six-well dishes. Plasmids encoding the EGFP and K2P channels were cotransfected with Lipofectamine 3000 reagent (Invitrogen) according to the manufacturer's instructions.

Electrophysiological Recording. To measure the currents of the K2P channels expressed in CHO-K1 cells, standard whole-cell patch-clamping experiments were performed at room temperature. Pipettes with resistance ranging from 2.0 to $5.0 \mathrm{M} \Omega$ were pulled using borosilicate glass capillaries (World Precision Instruments). During the recording process, constant perfusion of the bath solution was maintained using a BPS perfusion system (ALA Scientific). The pipette solution contained the following: $145 \mathrm{mM} \mathrm{KCl,} 1 \mathrm{mM} \mathrm{MgCl} 2$, $5 \mathrm{mM}$ EGTA, and $10 \mathrm{mM}$ HEPES (pH 7.3 with $\mathrm{KOH}$ ). The bath solution contained the following: $140 \mathrm{mM} \mathrm{NaCl}, 5 \mathrm{mM} \mathrm{KCl}, 2 \mathrm{mM}$ $\mathrm{CaCl} 2,1 \mathrm{mM} \mathrm{MgCl} 2,10 \mathrm{mM}$ glucose, and $10 \mathrm{mM}$ HEPES ( $\mathrm{pH} 7.4$ with $\mathrm{NaOH})$.

The whole-cell currents were recorded using an EPC-10 amplifier (HEKA), and signals were filtered at $2 \mathrm{kHz}$, digitized using a DigiData 1440A, and analyzed with pClamp 9.2 software (Molecular Devices). The series resistance was compensated by $60 \%$.

Homology Modeling. The structures of the TASK-3 channel were constructed on the basis of the crystal structures of the TREK-2 channel (PDB code 4BW5 and 4XDJ, identity: 31\%) by using Modeler (Sali and Blundell, 1993) and were reported separately by their conductive and nonconductive states. Multiple sequence alignment was generated by using the Clustal Omega web server (https:// www.ebi.ac.uk/Tools/msa/clustalo).

Statistics. Patch-clamp data were processed using Clampfit 10.2 (Molecular Devices) and then analyzed in GraphPad Prism 5 (GraphPad Software). Dose-response curves were fitted with the Hill equation, $\mathrm{E}=\mathrm{E}_{\max } /\left[1-\left(\mathrm{EC}_{50} / \mathrm{C}\right]^{\mathrm{P}}\right)$, where $\mathrm{EC}_{50}$ is the drug concentration producing the half-maximum response. The data are shown as the means \pm S.E.M., and the significance was estimated using one-way analysis of variance followed by Dunnett's post-hoc test. Statistical significance: ${ }^{*} P<0.05$, ** $P<0.01$, *** $P<0.001$.

\section{Results}

Identification of NPBA as a Selective TASK-3 Activator. To address the lack of selective pharmacology tools for the TASK-3 channel, we developed an HTS to discover selective small-molecule TASK-3 modulators. Before the HTS, HEK293 cell lines stably expressing human TASK-3 or its closest homolog TASK-1 were established, because selective active compounds for the TASK subfamily would have a greater tendency to exhibit selectivity among the K2P family. Then, the channel activity was measured by a thallium flux assay as in previous work (Yu et al., 2015; Yue et al., 2016).

Within the project, more than 300,000 compounds were screened, of which 1417 compounds were identified as TASK3 activators. Among these active compounds, N-(2-((4-nitro2-(trifluoromethyl)phenyl)amino)ethyl)benzamide (NPBA) 
A

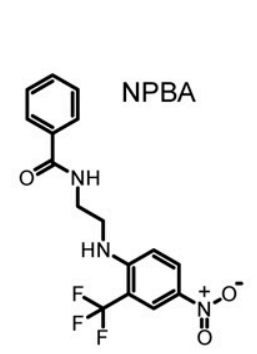

D

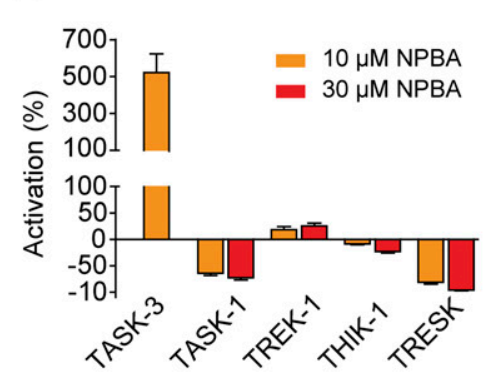

B

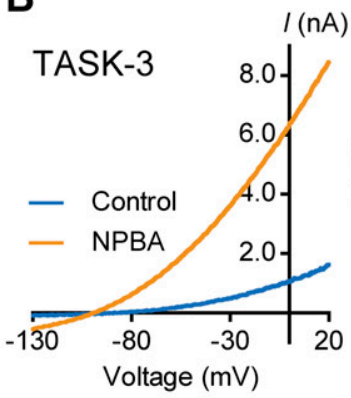

E

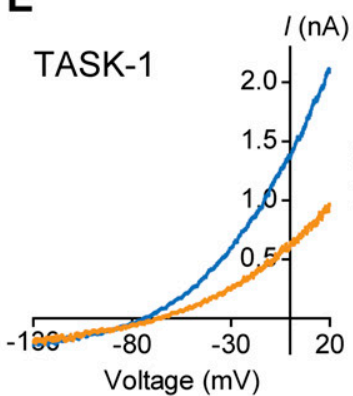

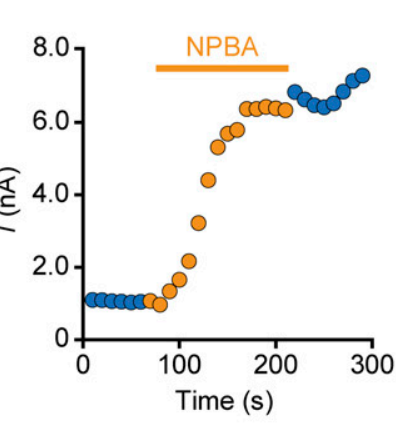
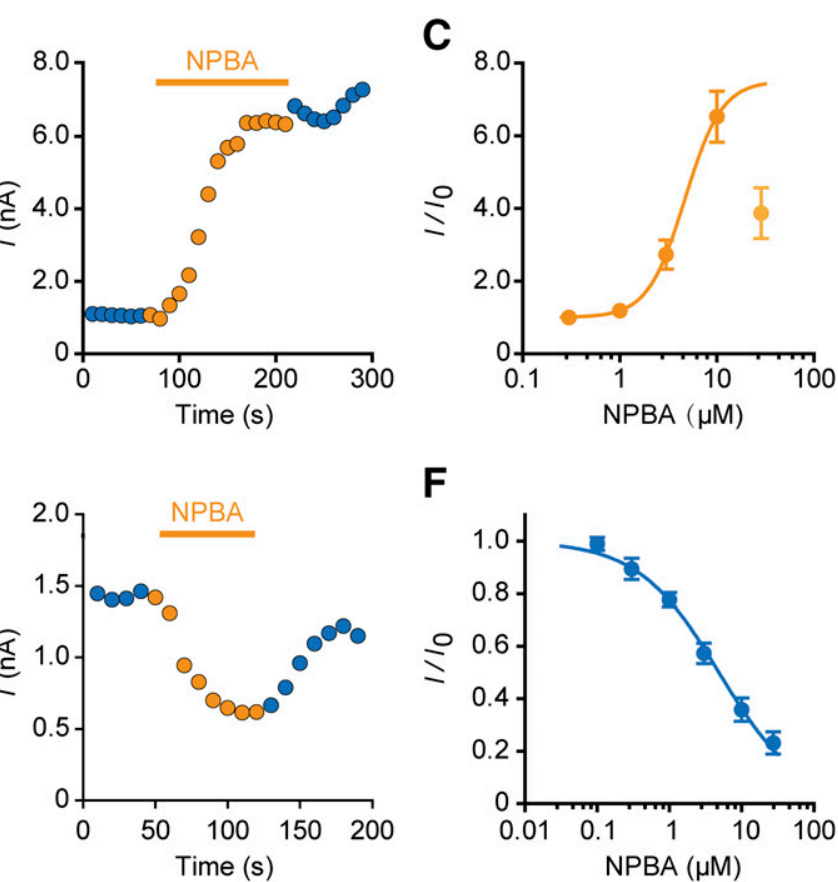

$\mathbf{F}$

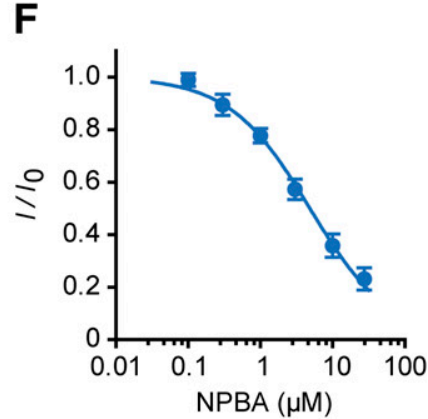

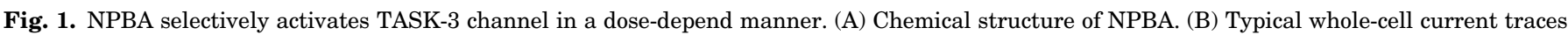

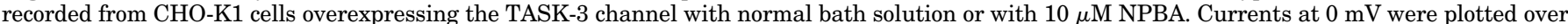

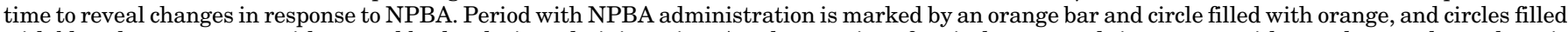

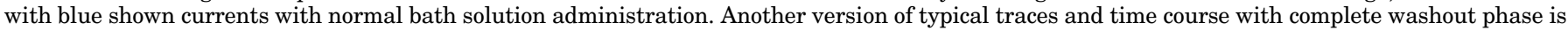

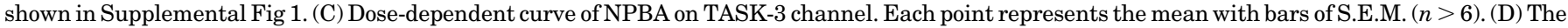

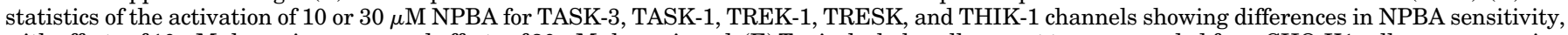

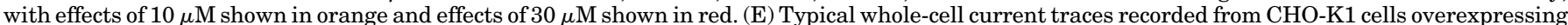

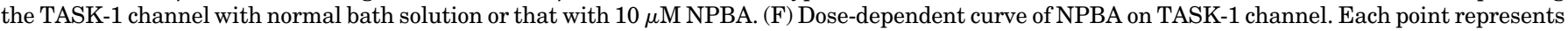
the mean with bars of S.E.M. $(n>4)$.

exhibited the best potency for TASK-3 activation and showed good selectivity between TASK channels. Figure 1A shows the molecular structure of NPBA.

To confirm the activation of NPBA and to estimate its potency, whole-cell patch-clamp recordings were performed. CHO-K1 cells transiently transfected with wild-type human TASK-3 or other K2P channels were used for the recording, and we ran a voltage-ramp protocol from -130 to $20 \mathrm{mV}$ to obtain the current-voltage characteristic (I-V) curve. The current at $0 \mathrm{mV}$ was chosen to measure the potency of NPBA toward different channels. For the dose-response curve study, we tested concentrations of NPBA ranging from 0.1 to $30 \mu \mathrm{M}$, as the compound cannot be completely dissolved at a higher concentration of $100 \mu \mathrm{M}$. NPBA showed reversible dosedependent activation of TASK-3 whole-cell currents with an $\mathrm{EC}_{50}$ of $6.7 \mu \mathrm{M}$, and the currents were increased by up to approx. 6-fold at a concentration of $10 \mu \mathrm{M}$ (Fig. 1, B and C). We also investigated the effects of $10 \mu \mathrm{M}$ NPBA on other K2Ps, including TASK-1, TREK-1, TRESK, and THIK-1, but no activation was detected in these channels (Fig. 1D), suggesting excellent selectivity of NPBA toward TASK-3 channels. As the closest homolog of TASK-3, TASK-1 was also tested in a dose-response curve study, and it was reversibly inhibited by NPBA in a dose-dependent manner, with an $\mathrm{IC}_{50}$ of 7.5 $\mu \mathrm{M}$ (Fig. 1, E and F). These results indicated that NPBA was a potent activator of the TASK-3 channel and has good selectivity.

The A108 Residue Located at the Extracellular End of TM2 Is Crucial for Activation by NPBA. To identify regions of the TASK-3 channel that are necessary for activation by NPBA, TASK-3 and TASK-1 subunits were used to make chimeric constructs because they shared highly homologous sequences but showed greatly different responses to NPBA.

Using In-Fusion cloning technology, we first replaced the N-terminus of TASK-3 with a homologous sequence from TASK1 and gradually extended the replaced region. We constructed T1-85-T3, in which amino acids 1-85 of TASK-3 were replaced by the corresponding part of TASK-1, and we constructed T1131-T3, T1-169-T3, and T1-209-T3 in the same manner. Then, the effects of NPBA on these chimeras were tested at a concentration of $10 \mu \mathrm{M}$. The first chimera, T1-85-T3, with the junction in the first pore domain, retained the activation by NPBA (Fig. 2A), whereas the other chimeras with the junction at or after position 131 in this series were not activated by NPBA (Fig. 2, B-D). These results indicated that there were key NPBA determinants within amino acids 85-131.

A mutagenesis scan was then performed to investigate the critical residues in this region. We tested the effects of swapping each of the amino acids that differed between TASK3 and TASK-1 within section 85-131 (Fig. 2E). In the TASK-3 channel, these four amino acids were individually replaced by those in the homologous sites of TASK-1. Among the four TASK-3 mutations (Fig. 2E), A105G moderately impaired the activation by NPBA, and the current was increased by approx. 3-fold under $10 \mu \mathrm{M}$ NPBA (Figs. 2F and 4G), while A108V completely abrogated the activation by NPBA and showed inhibition at $10 \mu \mathrm{M}$ NPBA (Figs. 2G and 4G). However, the other two mutants, G102S and V115L, showed an NPBA phenotype that was indistinguishable from the wild type (Fig. 4G). 
A

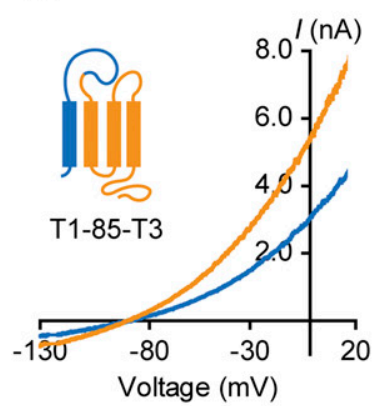

C

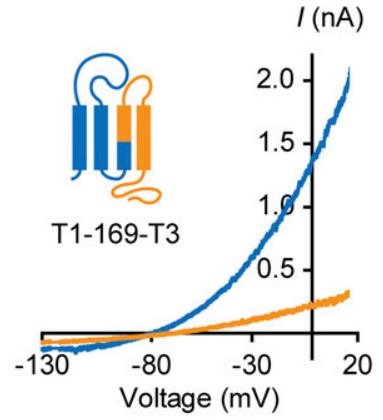

E
B
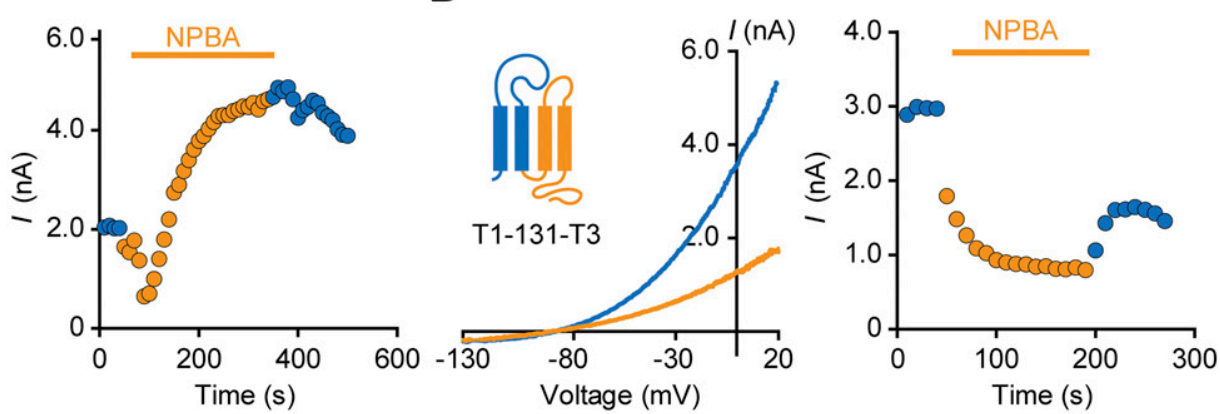

D
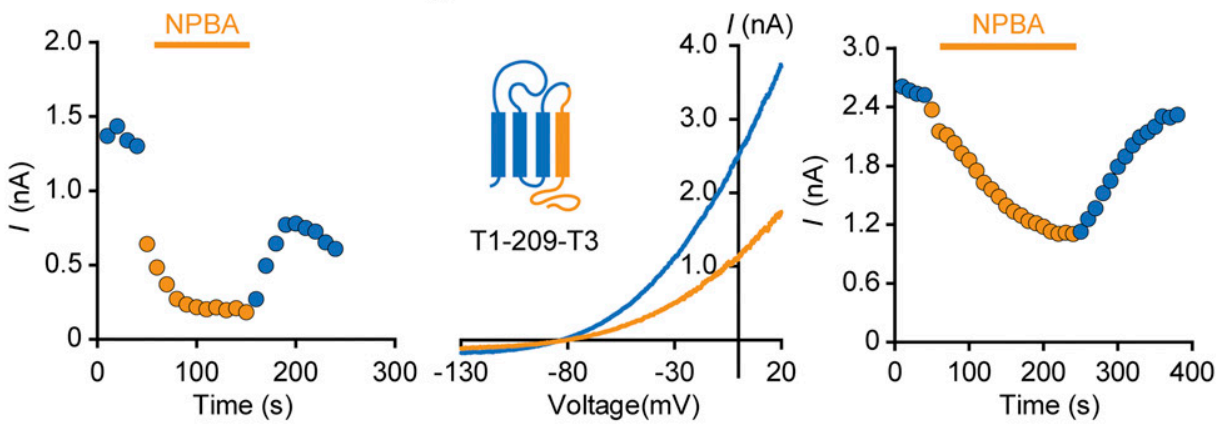

$\begin{array}{lc}105 & 108 \\ \downarrow & \downarrow\end{array}$

TM2
$\mathbf{F}$

TASK-3 (A105G)

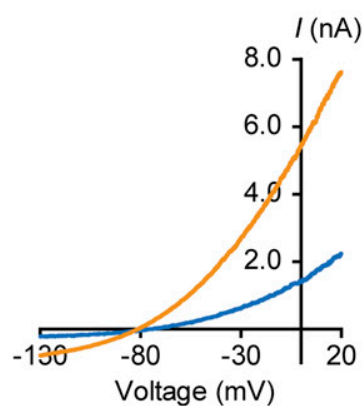

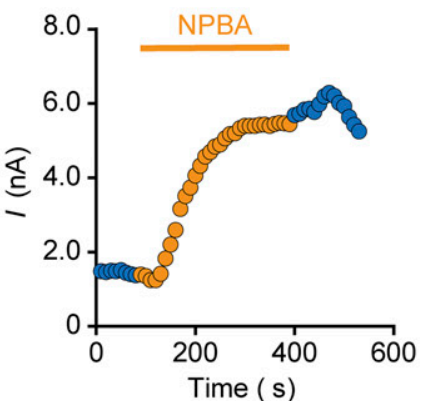

G

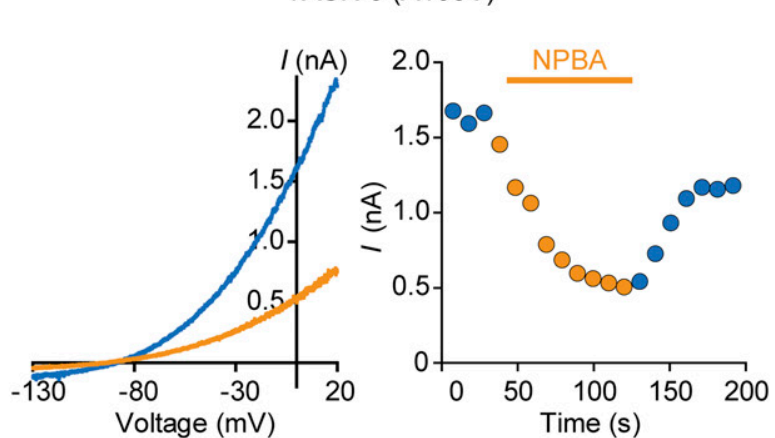

Fig. 2. Segment 85-131 is important for TASK-3 activation. (A-D) Typical whole-cell current traces and time courses recorded from CHO-K1 cells overexpressing the T1-85-T3 (A), T1-131-T3 (B), T1-169-T3 (C), or T1-240-T3 (D) mutant channel with $10 \mu \mathrm{M}$ NPBA. Protein secondary structure of chimera is shown beside the current traces, and section of TASK-3 appears in orange whereas section of TASK-1 appears in blue. (E) Sequence alignment of TASK-3 and TASK-1 for the residues 85-131. Residues different in TASK channels appear in jacinth. The pore domain and TM2 are marked above the sequences. Residues revealed important in swapping mutagenesis are marked with arrows. (F and G) Typical whole-cell current traces and time courses recorded from CHO-K1 cells overexpressing the TASK-3(A105G) (F) or TASK-3(A108V) (G) mutant with $10 \mu \mathrm{M}$ NPBA.

Since the alanine at position 108 (A108) seemed to be crucial for activation by NPBA, we replaced this alanine with various other amino acids, including phenylalanine, leucine, isoleucine, glycine, tyrosine, cysteine, and serine. Among all these A108 mutants, only A108G or A108S showed current activation in response to $10 \mu \mathrm{M}$ NPBA, whereas all other mutants showed the same current inhibition as A108V (Fig. 3A). Furthermore, we performed alanine scanning mutagenesis within the residues around A108 and evaluated the responses under $10 \mu \mathrm{M}$ NPBA. Among the nine mutant channels, four mutations (T103A, D104A, F109A, and M111A) eliminated activation by NPBA and showed current inhibition by NPBA, whereas mutations of the remaining five residues (P101A, G102A, G106A, K107A, and C110A) showed only a slight decrease in the activation degree resulting from NPBA (Fig. 3B). Taken together, these findings suggested that the residues around A108 played a crucial role in NPBA activation on the TASK-3 channel. 
A

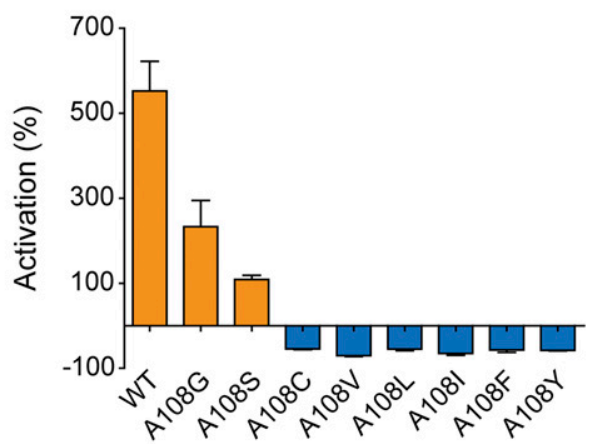

C

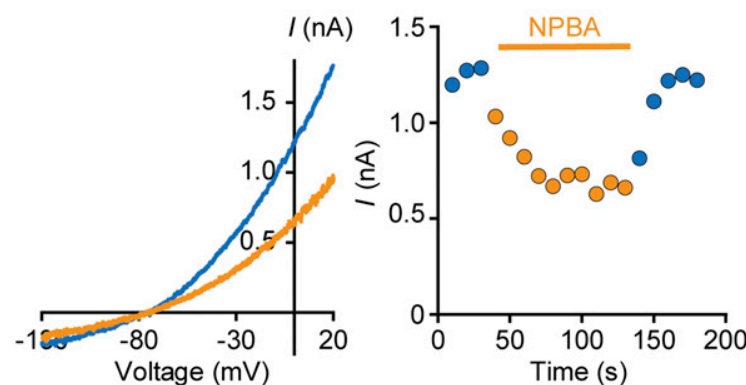

B

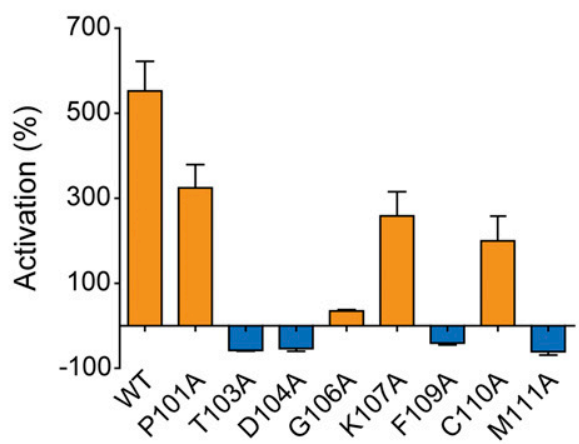

D

TASK-1(G105A, V108A)

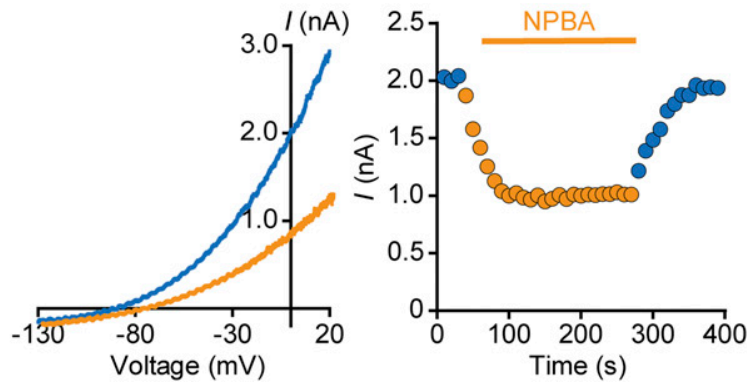

Fig. 3. Residues around A108 is important for NPBA activation. (A) The statistics of the activation of $10 \mu \mathrm{M}$ NPBA for wild-type TASK-3 ( $n=10)$, A108G $(n=4), \mathrm{A} 108 \mathrm{~S}(n=4), \mathrm{A} 108 \mathrm{C}(n=4), \mathrm{A} 108 \mathrm{~V}(n=7), \mathrm{A} 108 \mathrm{~L}(n=4), \mathrm{A} 108 \mathrm{I}(n=4), \mathrm{A} 108 \mathrm{~F}(n=4)$, and A108Y $(n=4)$. (B) The statistics of the activation of $10 \mu \mathrm{M}$ NPBA for wild-type TASK-3 $(n=10), \mathrm{P} 101 \mathrm{~A}(n=4)$, T103A $(n=5), \mathrm{D} 104 \mathrm{~A}(n=5), \mathrm{G} 106 \mathrm{~A}(n=4), \mathrm{K} 107 \mathrm{~A}(n=6), \mathrm{F} 109 \mathrm{~A}(n=4), \mathrm{C} 110 \mathrm{~A}(n=4)$, and $\mathrm{M} 111 \mathrm{~A}(n=5)$, with activation effects shown in orange and inhibition effects shown in blue. (C and D) Typical whole-cell current traces and time courses recorded from CHO-K1 cells overexpressing the TASK-1(V108A) (C) or TASK-1(V108A, G105A) (D) mutant with $10 \mu \mathrm{M}$ NPBA.

A TASK-1 Mutant with Alanine at Position 108 Resists NPBA Activation. Since the TASK-3 mutant A108V showed a total loss of NPBA activation, we wondered whether the corresponding TASK-1 mutant V108A would gain activation by NPBA. As shown in Fig. 3C, however, TASK-1(V108A) could not be activated by $10 \mu \mathrm{M}$ NPBA. A double-mutant TASK-1 channel (G105A, V108A) was then constructed by introducing G105A into TASK-1(V108A). We found that the newly constructed mutant channel could not be activated by NPBA (Fig. 3D). In contrast, it showed an inhibition similar to that in the wild-type TASK-1 or TASK-1(V108A). These results implied that these residues on TM2 were not the only determinants responsible for the different NPBA phenotypes of TASK- 1 and TASK-3.

The Intracellular End of TM3 Is Important for NPBA Activation. On the basis of the results above, we concluded that there should be other important residues for NPBA activation in addition to A105 and A108. To identify these unknown determinants, we constructed another series of chimeras. In this group of chimeras, the C-terminal of TASK-3 was replaced by the homologous sequence of TASK1 , and the junction was moved forward gradually. We constructed T3-240-T1 as the protein sequence after position 240 of TASK-3 was replaced by the corresponding section of TASK-1 and constructed T3-209-T1, T3-169-T1, and T3-131T1 in the same manner. We then tested the effects of NPBA at a $10 \mu \mathrm{M}$ concentration. With the junction moving forward, T3-240-T1, T3-209-T1, and T3-169-T1 could all be activated by NPBA (Fig. 4, A-C), whereas the last chimera T3-131-T1 could not be activated significantly by $10 \mu \mathrm{M}$ NPBA (Fig. 4D). These results suggested that there were determinants for NPBA activation within section 131-169 in addition to 85-131.

Next, we performed scanning mutagenesis within amino acids 131-169 of TASK-3, and 16 mutants were constructed and evaluated (Fig. 4, E and G). Among these mutations, the mutation E157A weakened NPBA activation the most, and the mutant current showed only a 2 -fold enhancement under $10 \mu \mathrm{M}$ NPBA (Fig. 4F). There are also other mutations that impaired the activation, such as M168I and $\mathrm{K} 141 \mathrm{H}$, which showed a moderate approx. 3-fold current activation in response to $10 \mu \mathrm{M}$ NPBA (Fig. 4G).

Since the glutamic acid at position 157 (E157) seems to be important for NPBA activation, we further changed this negatively charged amino acid to negatively charged aspartic acid, positively charged arginine, or neutral glutamine and determined the effects of $10 \mu \mathrm{M}$ NPBA on these mutants. Both E157D and E157R showed the same NPBA phenotype as E157A, with only a 2-fold current enhancement under $10 \mu \mathrm{M}$ NPBA. However, the E157Q current was activated by $10 \mu \mathrm{M}$ NPBA as effectively as the wild-type TASK-3 channel, showing 6-fold activation under $10 \mu \mathrm{M}$ NPBA (Fig. 5A). These results suggested that $\mathrm{E} 157$ is also an important residue for NPBA activation, and the volume rather than the charge of the residue at position 157 is the key characteristic necessary to achieve a potent activation by NPBA.

NPBA Activates TASK-3 through a Mechanism Different from Inhalation Anesthetics. E157 was predicted to be located in the intracellular end of TM3 on the basis of hydropathic analysis (Kim et al., 2000) and is very close to M159 (Fig. 4E; Fig. 6G), a residue reported to be the binding 
A

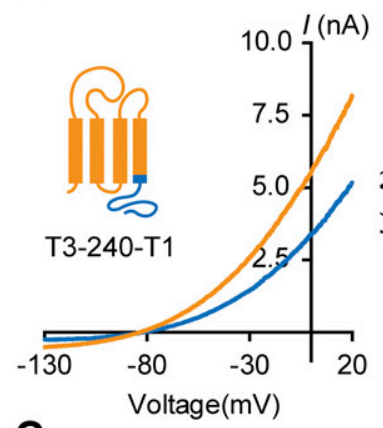

C

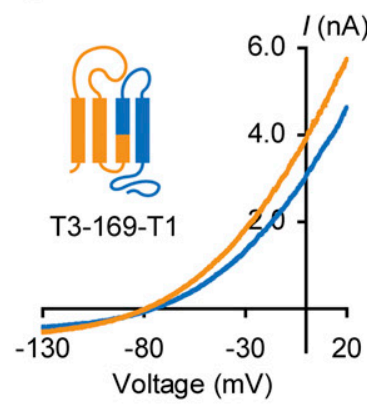

E
B
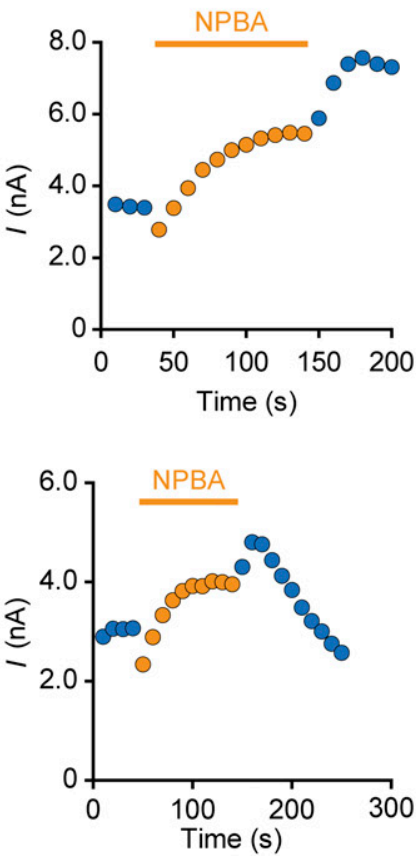

D
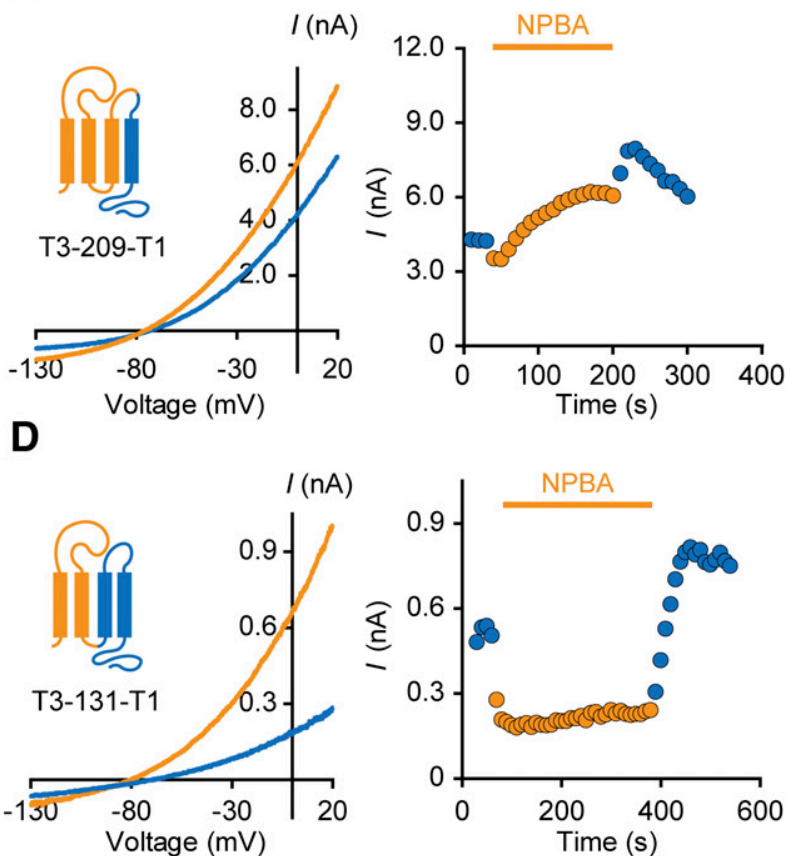

131 - R I N T L V R Y L L H R A K K G L G M R -150 $131-R M N T F V R Y L L K R$ I K K C C G M R -150

157

$\downarrow \quad \mathrm{TM} 3$

141 - R A D V S M A M V L I G F F S I S - 169

$141-N T D V S M E N$ M V T V G F F S M G - 169
$\mathbf{F}$

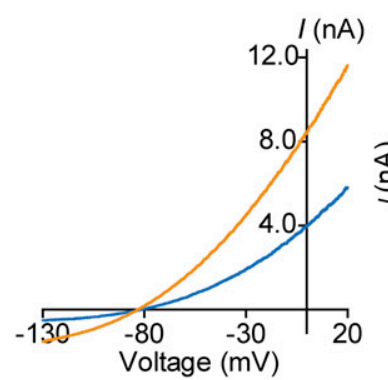

TASK-3(E157A)

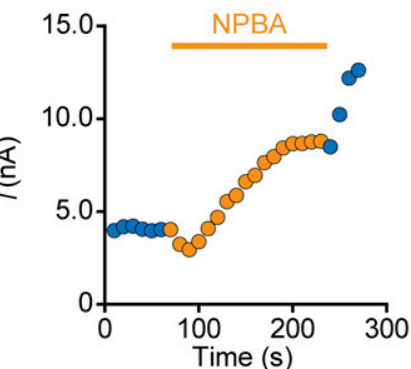

G

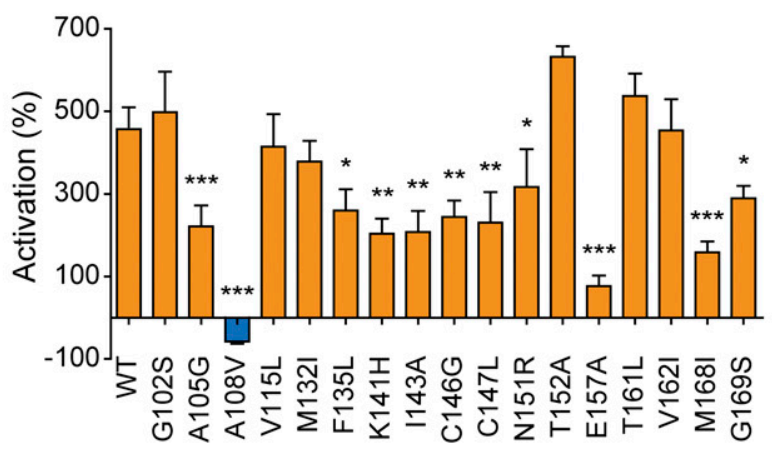

Fig. 4. Segment 131-169 is important for NPBA activation. (A-D) Typical whole-cell current traces and time courses recorded from CHO-K1 cells overexpressing the T3-240-T1 (A), T3-169-T1 (B), T3-131-T1 (C), or T3-131-T1 (D) mutant channel with $10 \mu \mathrm{M}$ NPBA. Protein secondary structure of chimera is shown beside the current traces, and section of TASK-3 appears in orange and section of TASK-1 appears in blue. (E) Sequence alignment of TASK-3 and TASK-1 for the residues 131-169. Residues different in TASK channels appear in jacinth. The TM3 is marked above the sequences. Residues revealed important in swapping mutagenesis are marked with arrows. (F) Typical whole-cell current traces and time courses recorded from CHO-K1 cells overexpressing the TASK-3(E157A) mutant with $10 \mu \mathrm{M}$ NPBA. (G) Histograms summarizing the activation of $10 \mu \mathrm{M}$ NPBA for wild-type TASK-3 and TASK-3 mutants with substitution of TASK-1 residues within 85-131 or 131-169, with activation effects shown in orange and inhibition effects shown in blue. A108V is the only mutant showing a final inhibition with $10 \mu \mathrm{M}$ NPBA. Statistical significance: $* P<0.05, * * P<0.01, * * * P<0.001$. The baseline and NPBA peak-activated current values and the I/I0 of TASK-3 mutants is shown in Supplemental Table 1.

site of nonselective inhalation anesthetics. Thus, we wondered whether activation by NPBA and activation by inhalation anesthetics occurred through a common mechanism. In this study, chloroform was selected as a representative inhalation anesthetic and increased the wild-type TASK-3 current approx. 2-fold at a concentration of $5 \mathrm{mM}$ (Fig. 5, B and J), which is consistent with previous results (Andres-Enguix et al., 2007). Thereafter, we determined and compared the effects of NPBA and chloroform on the mutant channels that have been reported to show impaired anesthetic activation. First, we constructed the mutants that showed increased basal currents and resistance to activation by inhalation 
A

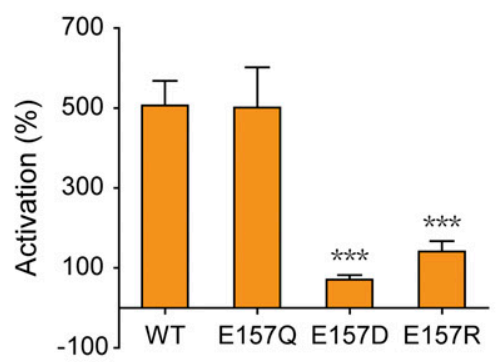

C

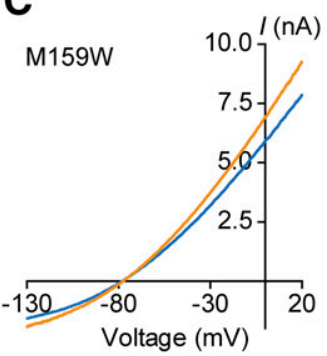

E

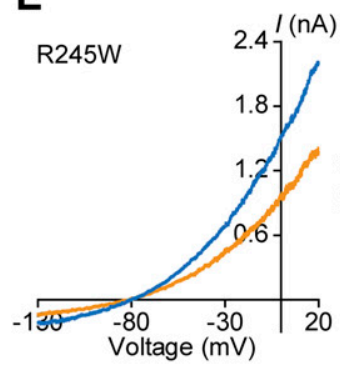

G

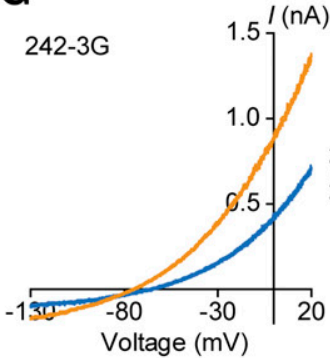

I

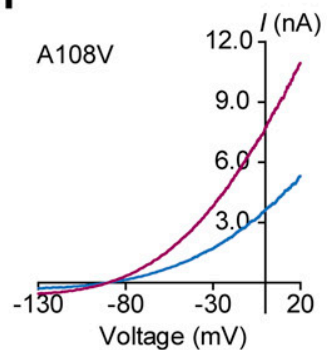

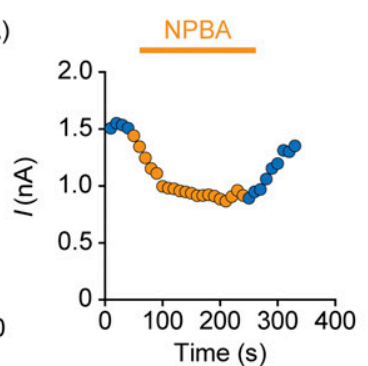
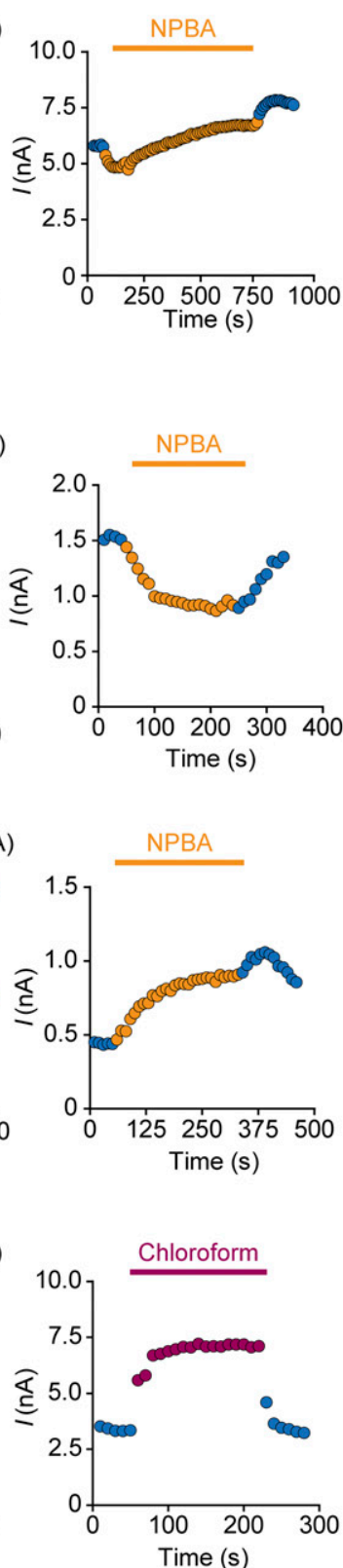

B
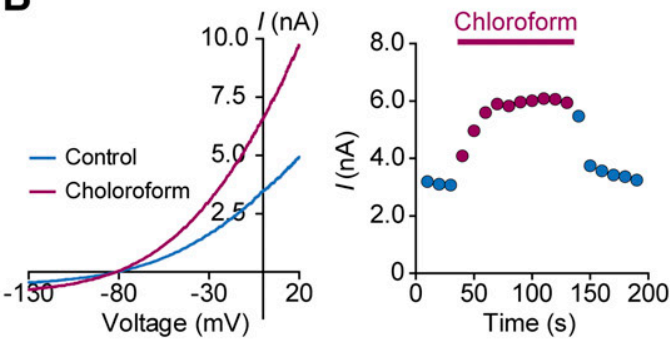

D
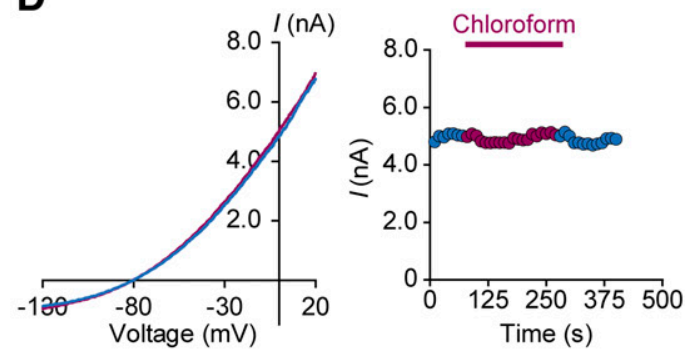

F
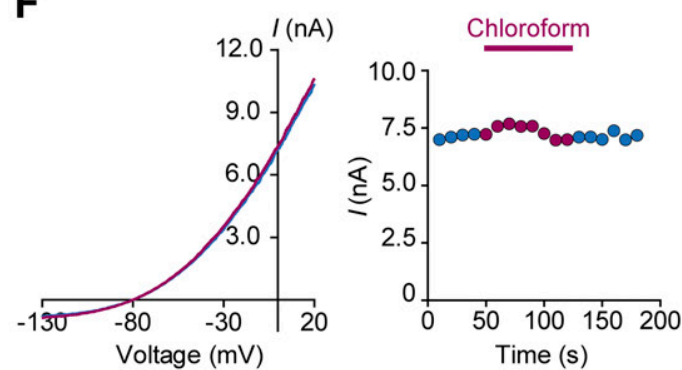

H
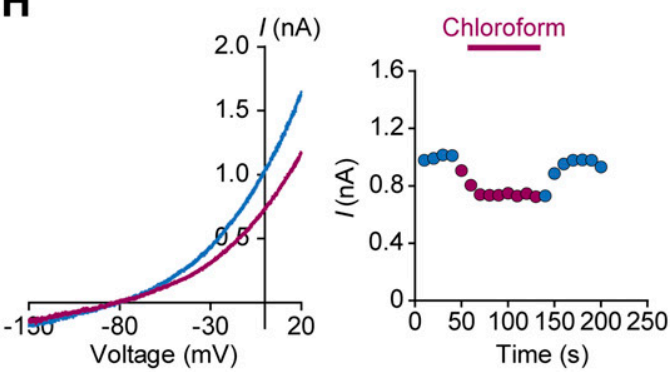

J

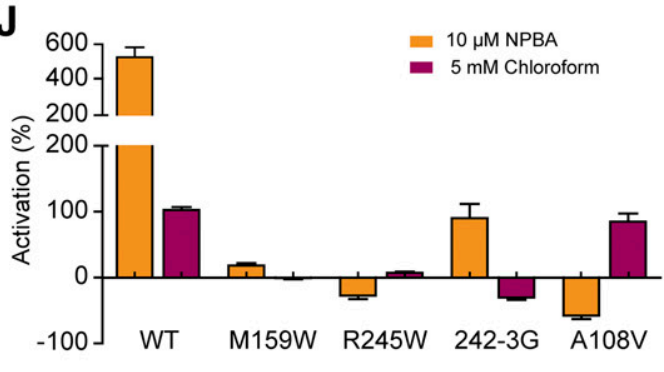

Fig. 5. Activations by NPBA and chloroform have different determinants. (A) The statistics of the activation of $10 \mu \mathrm{M}$ NPBA for wild-type TASK-3 $(n=10), \mathrm{E} 157 \mathrm{Q}(n=4), \mathrm{E} 157 \mathrm{D}(n=5)$, and E157R $(n=5)$. The activation of NPBA on TASK-3 mutants was compared with wild type, statistical significance: $* P<0.05$; ** $P<0.01$; *** $P<0.001$. (B) Typical whole-cell current traces and time courses recorded from CHO-K1 cells overexpressing TASK-3 channel with $5 \mathrm{mM}$ chloroform. (C and D) Typical whole-cell current traces and time courses recorded from CHO-K1 cells overexpressing the TASK-3(M159W) mutant with $10 \mu \mathrm{M}$ NPBA (C) or $5 \mathrm{mM}$ chloroform. (E and F) Typical whole-cell current traces and time courses recorded from CHO-K1 cells overexpressing the TASK-3(R245W) mutant with $10 \mu \mathrm{M}$ NPBA $(\mathrm{E})$ or $5 \mathrm{mM}$ chloroform. (G and H) Typical whole-cell current traces and time courses recorded from CHO-K1 cells overexpressing the 242-3G mutant with $10 \mu \mathrm{M}$ NPBA (G) or $5 \mathrm{mM}$ chloroform (H). (I) Typical whole-cell current traces and time courses recorded from CHO-K1 cells overexpressing the A108V mutant with $5 \mathrm{mM}$ chloroform. Magenta trace, current under $5 \mathrm{mM}$ chloroform; blue trace, current under normal bath solution. Period with chloroform administration is marked by a magenta bar and circle filled with magenta, and circles filled with blue show currents with normal bath solution administration. (J) Histograms summarizing the activation of $10 \mu \mathrm{M}$ NPBA or $5 \mathrm{mM}$ chloroform for wild-type TASK-3, M159W, R245W, 242-3G, and A108V, with NPBA effects shown in bars filled with orange and chloroform shown with bars filled with magenta. $n=4-10$. 
A

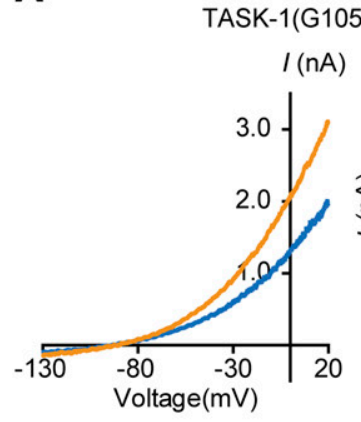

C
B

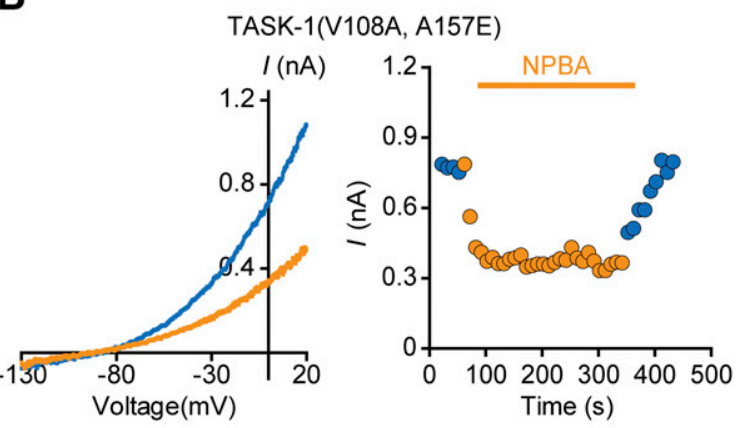

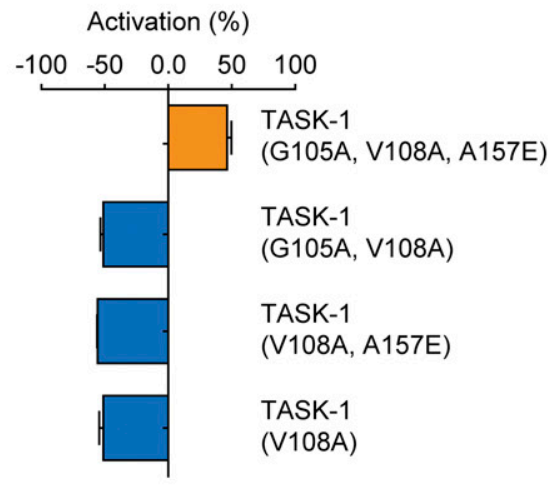

E

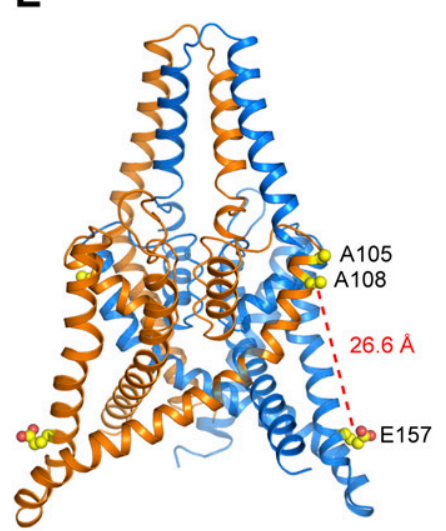

F

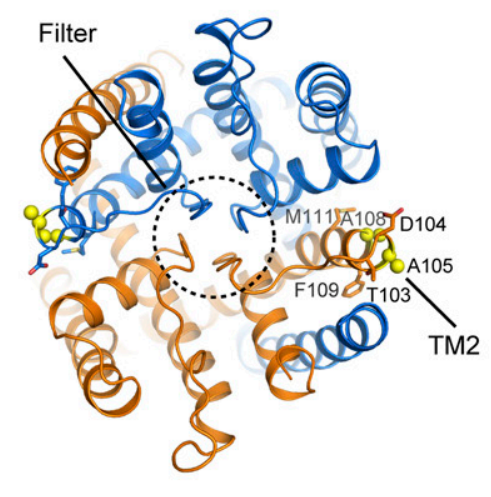

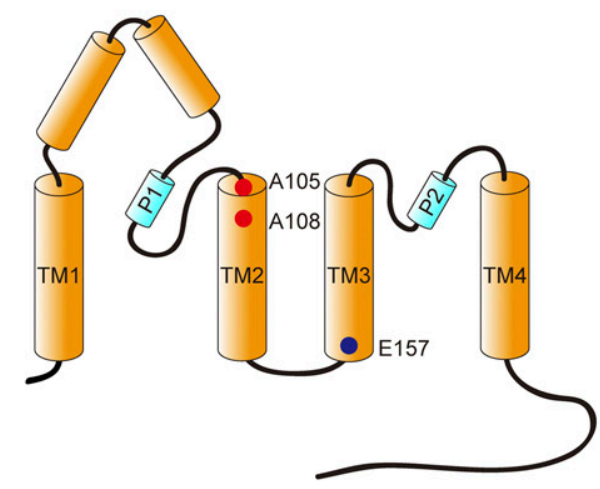

G

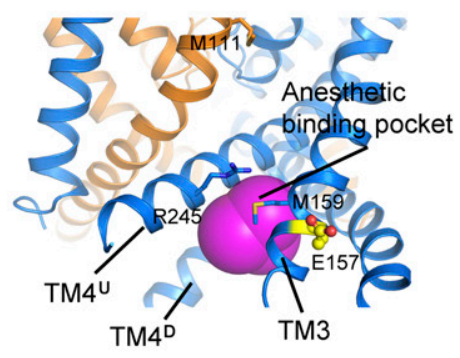

Fig. 6. A TASK-1 mutant gains activation by NPBA after A105, A108, and E157 transplanted into it. (A and B) Typical whole-cell current traces and time courses recorded from CHO-K1 cells overexpressing TASK-1(V108A, G105A, A157E) (A) and TASK-1(V108A, A157E) (B) channels with 10 $\mu$ M NPBA. (C) Histograms summarizing the activation of $10 \mu \mathrm{M}$ NPBA TASK-1(V108A, G105A, A157E) $(n=8)$, TASK-1(V108A, A157E) ( $n=6)$, and TASK-1(V108A, G105A) $(n=5)$. (D) Important residues for NPBA activation in a topology of TASK-3 subunit. (E) Homology model of TASK-3 channel. Crucial residues A105, A108, and E157 for NPBA activation are shown in yellow spheres. (F) Top view of TMs and filter domain, the important residues on one TM2 subunit are labeled. (G) Detailed view of protein domains around E157. The putative anesthetic binding pocket is shown in purple spheres. The up and down states of distal end of TM4 are represented by TM $4^{\mathrm{U}}$ and TM $4^{\mathrm{D}}$ separately.

anesthetics (Conway and Cotten, 2012). M159W completely abrogated the activation by $5 \mathrm{mM}$ chloroform, and activation by NPBA was partially retained (Fig. $5, \mathrm{C}, \mathrm{D}$, and J). Then, we constructed the mutant R245W with a large tryptophan predicted to occupy the space around M159, similar to M159W (Fig. 6G). Notably, this alteration abolished activation by both NPBA and chloroform and showed a different NPBA phenotype from that of M159W (Fig. 5, E, F, and J). In addition, we constructed a TASK-3 mutant 242-3G with three residues, V242-V243-L244, replaced by glycines, on the basis of a $\mathrm{K} 2 \mathrm{P} 2.1-3 \mathrm{G}$ mutant predicted to uncouple the cross-talk between the pore and carboxyl terminus (Bagriantsev et al., 2012). Subsequently, $5 \mathrm{mM}$ chloroform failed to induce an increase in current in the 242-3G mutant and instead showed an inhibitory effect on the current (Fig. 5, $\mathrm{H}$ and J), suggesting that this triple glycine mutation also affects the TASK-3 channel. However, the activation by NPBA was retained in the 242-3G mutant, with a potency of 2-fold under a concentration of $10 \mathrm{mM}$ (Fig. 5, G and J). Finally, we tested the effect of $5 \mathrm{mM}$ chloroform on the A108V mutant that completely abolished NPBA activation (Fig. 2G). The activation degree of $5 \mathrm{mM}$ chloroform for $\mathrm{A} 108 \mathrm{~V}$ was indistinguishable from that for the 
wild-type TASK-3 channel (Fig. 5, I and J). Taken together, these results demonstrated that though activation by NPBA and inhalation anesthetics shared some key residues, such as M159, they probably activate the channel through different action mechanisms.

A TASK-1 Mutant with Three Key Residues Swapped Gains NPBA Activation. Since the amino acids 131-169 were also shown to be vital regions for NPBA activation and E157 seemed to be the most important residue in this region, we transplanted E157 into the mutant channel TASK1(G105A, V108A) to construct the mutant TASK-1(G105A, $\mathrm{V} 108 \mathrm{~A}, \mathrm{~A} 157 \mathrm{E})$, and then we determined the effect of $10 \mu \mathrm{M}$ NPBA on this triple-mutant channel. The logic here was to try to construct a TASK-1 mutant that could be activated by NPBA to confirm the importance of these residues. Dramatically, TASK-1(G105A, V108A, A157E) gained NPBA activation, and the current in the mutant showed a 1.5-fold increase under an NPBA concentration of $10 \mu \mathrm{M}$ (Fig. 6, A and C). In contrast, a mutant with two sites changed, TASK-1(V108A, A157E) did not gain activation by $10 \mu \mathrm{M}$ NPBA (Fig. 6, B and C). Notably, TASK-1(G105A, V108A, A157E) showed an NPBA phenotype combining the responses of TASK-1 and TASK-3. After the administration of NPBA, the mutant current decreased initially for seconds and then increased gradually, displaying an inward hook on the time course plot (Fig. 6A). We speculated that the intrinsic inhibition of TASK1 perhaps counteracts the acquired potentiation from the three mutations (G105A, V108A, A157E), resulting in a weaker activation of the current amplitude than that of the wild-type TASK-3 channel. These results demonstrated that A108, A105, and E157 were important for activation (Fig. 6D).

On the basis of the crystal structures of TREK-2, we created an open-state homology model of TASK-3 (Fig. 6E). Structurally, the key residues A105 and A108 lie at the extracellular end of TM2, a pore-lining helix adjacent to P1 and P2 (Fig. 6 , D-F), whereas the third key residue E157 is located in the intracellular end of TM3 (Fig. 6, D, E, and G). Notably, these two regions are separated by a long distance of approx. $26.6 \AA$ (Fig. 6E).

\section{Discussion}

Among K2P channels, the TASK-3 channel has been implicated in a number of normal physiologic processes or disorders, but few selective TASK-3 channel modulators have been identified, which hinders the understanding of channel function in physiology. Terbinafine, an antifungal medication, was the first and previously the only selective activator of TASK-3 reported, but this compound only has a moderate activation effect. In the present work, we identified NPBA as a novel agonist of the TASK-3 channel by HTS. NPBA produces approx. 6-fold activation under a concentration of $10 \mu \mathrm{M}$ (Fig. $1 \mathrm{~B})$ and is thus more potent than terbinafine, though the $\mathrm{EC}_{50}$ of NPBA $(6.7 \mu \mathrm{M})$ is almost equal to that of terbinafine $(6.2$ $\mu \mathrm{M}$ ) (Fig. 1C). In addition, NPBA also has good selectivity (Fig. 1C), which makes it a useful pharmacological probe for in vitro studies of TASK-3 function and in further studies intended for therapeutic intervention.

The study of the molecular mechanisms of modulators is an important aspect of ion channel research. For TASK-3, previous studies mainly focused on the regulation of $\mathrm{pH}$ and inhalation anesthetics. Though some explicit results have been obtained (Andres-Enguix et al., 2007; Conway and Cotten, 2012), fewer novel elements in the activation mechanism of TASK-3 have been reported in recent years, which may be owing to the lack of novel modulators of TASK-3. Adopting the classic chimera strategy and various mutagenesis analyses in this work, we found several residues that are important for NPBA activation, whose alteration could significantly impair NPBA activation (Figs. 2-4). By transplanting three of these residues, A105, A108, and E157, into the TASK1 channel, which resists activation by NPBA, the TASK-1 mutant TASK-1(G105A, V108A, A157E) was constructed and dramatically gained activation by NPBA (Fig. 6, A and C). This gain of activation is more convincing because no binding assay or cocrystallization research had been performed in previous studies of TASK-3, which further confirms the importance of these residues. Among the three residues, A108 and A105 lie at the proximal amphipathic end of TM2 adjacent to P1 and P2 (Fig. 6, D and F), which may be related to selective filter gating, whereas E157 is spatially adjacent to the distal end of TM4, the up and down states of which have been proven to represent different conductive states (Fig. 6G) (Dong et al., 2015). These residues have never been mentioned in previous studies of TASK-3 and may provide clues for the understanding of the gate mechanism of TASK-3.

The mechanism of the activation of TASK-3 by inhalation anesthetics has been partially revealed. The binding site of these molecules was believed to be located on the intracellular side of TM3 near M159 (Andres-Enguix et al., 2007; Conway and Cotten, 2012). In addition, the amino acids 243-248 were also involved in the inhalation anesthetic activation (Talley and Bayliss, 2002). In the present work, by comparing the phenotypes of the response to NPBA and chloroform in multiple TASK-3 mutants, we found that some mutants, such as R245W, affected activations by both NPBA and chloroform (Fig. 5, E and F). However, the elements that are vital for the activation of NPBA and chloroform did not overlap perfectly. Specifically, the most crucial mutant for NPBA activation, A108V, did not change the chloroform activation (Fig. 2G; Fig. 5I); the mutant 242-3G retained NPBA activation, although chloroform activation was abolished (Fig. 5, G and $\mathrm{H})$. Among the mutations that weakened the activation by chloroform, M159W is a classic mutation that has proven to be effective by occupying the space around the binding site of chloroform with a tryptophan and mimicking a more conductive chloroform-bound conformation. Interestingly, R245W seems to affect the activation of chloroform in a similar manner since R245 is spatially near M159. However, NPBA activation was partially retained in M159W, suggesting that NPBA does not share M159 as a binding site with chloroform. Accordingly, the activation effect of NPBA on wild-type TASK3 was tested in the presence of $10 \mathrm{mM}$ chloroform, a saturated concentration of the inhalation anesthetic. We found that NPBA further significantly potentiated the increased current by chloroform (Supplemental Fig. 2). These results suggest that the novel activator NPBA may activate TASK-3 differently from inhalation anesthetics.

The TM4 and Ct domains in K2Ps have proven to be important regions for a range of signaling inputs within the cell, including temperature (Maingret et al., 2000), mechanical force (Patel et al., 1998), and phosphorylation (Bagriantsev et al., 2012). Notably, the crosstalk between the two regions can be broken by the 242-3G mutation (Bagriantsev et al., 2012). 
In our work, the 242-3G mutation completely abolished the activation by chloroform, which could be explained by the fact that activation by chloroform depends on the relay from the $\mathrm{Ct}$ domain to TM4 or that this $3 \mathrm{G}$ mutation causes conformational changes in the anesthetic pocket. However, NPBA activation was retained in $242-3 \mathrm{G}$, indicating that the binding site of NPBA is upstream of the link between TM4 and the Ct domain. Finally, A108, a brand new residue we identified, was found to be necessary only for activation by NPBA rather than chloroform. Because mutating three residues (G105A, V108A, and A157E) in TASK-1 partially conferred NPBA activation on TASK-1, we speculated that a long-range coupling between the extracellular segment around A108 and the intracellular domains around E157 might be involved in NPBA-induced activation. To elucidate the specific mechanism underlying potential cooperation, further studies, such as binding assays, computational simulations, and crystallization research, are necessary.

In conclusion, we have described the ability of a novel compound, NPBA, to activate the TASK-3 channel. This compound also displays marked specificity within the K2P group and may therefore be a good pharmacological tool for TASK-3 research. Several additional elements that are important for NPBA action have also been uncovered in our work. This finding provides a strategy to identify more novel TASK-3 activators, and the key residues we found, such as A108, may promote research on the gate mechanism of K2P channels.

\section{Authorship Contributions}

Participated in research design: Gao.

Conducted experiments: Tian, Lan.

Performed data analysis: Tian, Qiu, Lan, Li, Yang.

Wrote or contributed to the writing of the manuscript: Tian, Gao.

Note Added in Proof-The Significance Statement and National Natural Science Foundation of China grant number 61433017 were accidentally not included in the Fast Forward version published April 23, 2019. The Significance Statement has now been added, and the funding footnote has now been corrected.

\section{References}

Andres-Enguix I, Caley A, Yustos R, Schumacher MA, Spanu PD, Dickinson R, Maze M, and Franks NP (2007) Determinants of the anesthetic sensitivity of two-pore domain acid-sensitive potassium channels: molecular cloning of an anestheticactivated potassium channel from Lymnaea stagnalis. $J$ Biol Chem 282 : 20977-20990.

Bagriantsev SN, Clark KA, and Minor DL Jr (2012) Metabolic and thermal stimuli control K(2P)2.1 (TREK-1) through modular sensory and gating domains. EMBO J 31:3297-3308.

Bagriantsev SN, Peyronnet R, Clark KA, Honoré E, and Minor DL Jr (2011) Multiple modalities converge on a common gate to control K2P channel function. EMBO J 30:3594-3606.

Barel O, Shalev SA, Ofir R, Cohen A, Zlotogora J, Shorer Z, Mazor G, Finer G, Khateeb S, Zilberberg N, et al. (2008) Maternally inherited Birk Barel mental retardation dysmorphism syndrome caused by a mutation in the genomically imprinted potassium channel KCNK9. Am J Hum Genet 83:193-199.

Berg AP, Talley EM, Manger JP, and Bayliss DA (2004) Motoneurons express heteromeric TWIK-related acid-sensitive K+ (TASK) channels containing TASK-1 (KCNK3) and TASK-3 (KCNK9) subunits. J Neurosci 24:6693-6702.

Chemin J, Girard C, Duprat F, Lesage F, Romey G, and Lazdunski M (2003) Mechanisms underlying excitatory effects of group I metabotropic glutamate receptors via inhibition of $2 \mathrm{P}$ domain $\mathrm{K}+$ channels. EMBO J 22:5403-5411.

Conway KE and Cotten JF (2012) Covalent modification of a volatile anesthetic regulatory site activates TASK-3 (KCNK9) tandem-pore potassium channels. Mol Pharmacol 81:393-400.

Cotten JF, Keshavaprasad B, Laster MJ, Eger EI II, and Yost CS (2006) The ventilatory stimulant doxapram inhibits TASK tandem pore (K2P) potassium channel function but does not affect minimum alveolar anesthetic concentration. Anesth Analg 102:779-785.
Czirják G and Enyedi P (2002) Formation of functional heterodimers between the TASK-1 and TASK-3 two-pore domain potassium channel subunits. J Biol Chem 277:5426-5432.

Davies LA, Hu C, Guagliardo NA, Sen N, Chen X, Talley EM, Carey RM, Bayliss DA, and Barrett PQ (2008) TASK channel deletion in mice causes primary hyperaldosteronism. Proc Natl Acad Sci USA 105:2203-2208.

Dong YY, Pike AC, Mackenzie A, McClenaghan C, Aryal P, Dong L, Quigley A, Grieben M, Goubin S, Mukhopadhyay S, et al. (2015) K2P channel gating mechanisms revealed by structures of TREK-2 and a complex with Prozac. Science $\mathbf{3 4 7}$ 1256-1259.

Duprat F, Lesage F, Fink M, Reyes R, Heurteaux C, and Lazdunski M (1997) TASK, a human background $\mathrm{K}+$ channel to sense external $\mathrm{pH}$ variations near physiological pH. EMBO J 16:5464-5471.

Enyedi P and Czirják G (2010) Molecular background of leak K+ currents: two-pore domain potassium channels. Physiol Rev 90:559-605.

Goldstein SA, Bockenhauer D, O'Kelly I, and Zilberberg N (2001) Potassium leak channels and the KCNK family of two-P-domain subunits. Nat Rev Neurosci 2: 175-184

Gotter AL, Santarelli VP, Doran SM, Tannenbaum PL, Kraus RL, Rosahl TW, Meziane H, Montial M, Reiss DR, Wessner K, et al. (2011) TASK-3 as a potential antidepressant target. Brain Res 1416:69-79.

Guagliardo NA, Yao J, Hu C, Schertz EM, Tyson DA, Carey RM, Bayliss DA, and Barrett PQ (2012) TASK-3 channel deletion in mice recapitulates low-renin essential hypertension. Hypertension 59:999-1005.

Holter J, Carter D, Leresche N, Crunelli V, and Vincent P (2005) A TASK3 channel (KCNK9) mutation in a genetic model of absence epilepsy. J Mol Neurosci 25 : $37-51$

Kim D and Gnatenco C (2001) TASK-5, a new member of the tandem-pore $\mathrm{K}(+)$ channel family. Biochem Biophys Res Commun 284:923-930.

Kim Y, Bang H, and Kim D (2000) TASK-3, a new member of the tandem pore $\mathrm{K}(+)$ channel family. J Biol Chem 275:9340-9347.

Lesage F (2003) Pharmacology of neuronal background potassium channels. Neuropharmacology 44:1-7.

Linden AM, Sandu C, Aller MI, Vekovischeva OY, Rosenberg PH, Wisden W, and Korpi ER (2007) TASK-3 knockout mice exhibit exaggerated nocturnal activity, impairments in cognitive functions, and reduced sensitivity to inhalation anesthetics. J Pharmacol Exp Ther 323:924-934.

Maingret F, Lauritzen I, Patel AJ, Heurteaux C, Reyes R, Lesage F, Lazdunski M, and Honoré E (2000) TREK-1 is a heat-activated background K(+) channel. EMBO $J$ 19:2483-2491.

Mathie A (2007) Neuronal two-pore-domain potassium channels and their regulation by G protein-coupled receptors. $J$ Physiol 578:377-385.

Meadows HJ and Randall AD (2001) Functional characterisation of human TASK-3, an acid-sensitive two-pore domain potassium channel. Neuropharmacology 40: $551-559$.

Mu D, Chen L, Zhang X, See LH, Koch CM, Yen C, Tong JJ, Spiegel L, Nguyen KC, Servoss A, et al. (2003) Genomic amplification and oncogenic properties of the KCNK9 potassium channel gene. Cancer Cell 3:297-302.

Patel AJ, Honoré E, Lesage F, Fink M, Romey G, and Lazdunski M (1999) Inhalational anesthetics activate two-pore-domain background $\mathrm{K}+$ channels. Nat Neurosci 2:422-426.

Patel AJ, Honoré E, Maingret F, Lesage F, Fink M, Duprat F, and Lazdunski M (1998) A mammalian two pore domain mechano-gated S-like $\mathrm{K}+$ channel. EMBO J 17:4283-4290

Rajan S, Wischmeyer E, Xin Liu G, Preisig-Müller R, Daut J, Karschin A, and Derst C (2000) TASK-3, a novel tandem pore domain acid-sensitive $\mathrm{K}+$ channel. An extracellular histiding as pH sensor. J Biol Chem 275:16650-16657.

Sali A and Blundell TL (1993) Comparative protein modelling by satisfaction of spatial restraints. J Mol Biol 234:779-815.

Talley EM and Bayliss DA (2002) Modulation of TASK-1 (Kcnk3) and TASK-3 (Kcnk9) potassium channels: volatile anesthetics and neurotransmitters share a molecular site of action. J Biol Chem 277:17733-17742.

Talley EM, Sirois JE, Lei Q, and Bayliss DA (2003) Two-pore-Domain (KCNK) potassium channels: dynamic roles in neuronal function. Neuroscientist 9:46-56.

Talley EM, Solorzano G, Lei Q, Kim D, and Bayliss DA (2001) Cns distribution of members of the two-pore-domain (KCNK) potassium channel family. $J$ Neurosci 21:7491-7505

Wilke BU, Lindner M, Greifenberg L, Albus A, Kronimus Y, Bünemann M, Leitner MG, and Oliver D (2014) Diacylglycerol mediates regulation of TASK potassium channels by Gq-coupled receptors. Nat Commun 5:5540.

Wright PD, Veale EL, McCoull D, Tickle DC, Large JM, Ococks E, Gothard G, Kettleborough C, Mathie A, and Jerman J (2017) Terbinafine is a novel and selective activator of the two-pore domain potassium channel TASK3. Biochem Biophys Res Commun 493:444-450.

Yu H, Zou B, Wang X, and Li M (2015) Effect of tyrphostin AG879 on Kv 4.2 and Kv 4.3 potassium channels. Br J Pharmacol 172:3370-3382.

Yue JF, Qiao GH, Liu N, Nan FJ, and Gao ZB (2016) Novel KCNQ2 channel activators discovered using fluorescence-based and automated patch-clamp-based high-throughput screening techniques. Acta Pharmacol Sin 37:105-110.

Address correspondence to: Dr. Zhaobing Gao, CAS Key Laboratory of Receptor Research, State Key Laboratory of Drug Research, Shanghai Institute of Materia Medica, Chinese Academy of Sciences, Shanghai 201203, China. E-mail: zbgao@simm.ac.cn 